\title{
A Subband Approach to Channel Estimation and Equalization for DMT and OFDM Systems
}

\author{
Damián Marelli and Minyue Fu, Fellow, IEEE
}

\begin{abstract}
Cyclic prefix (CP) is commonly used for channel equalization of discrete multitone (DMT) and orthogonal frequency-division multiplexing (OFDM) systems. This is often done in conjunction with a time-domain equalizer (TEQ) for reducing the capacity overhead caused by the CP. However, the use of TEQ greatly increases the computational cost, and is unable to eliminate the need for the CP. In this paper, we propose a subband approach to channel estimation and channel equalization for DMT and OFDM systems. This approach involves splitting the received signals into a number of frequency bands (called subbands), and estimating a constant parameter in each subband. The subband approach is conceptually simple, requires no $\mathrm{CP}$, is much more numerically efficient than the TEQ method, and gives compatible or better estimation errors than the CP-based methods.
\end{abstract}

Index Terms-Discrete multitone (DMT), equalization, multicarrier, orthogonal frequency-division multiplexing (OFDM), subband adaptive filtering.

\section{INTRODUCTION}

D ISCRETE multitone (DMT) [1] and orthogonal frequencydivision multiplexing (OFDM) [2], [3] are multicarrier modulation techniques used in asymmetric digital subscriber line (ADSL) [4], high-speed digital subscriber loop (HDSL) ([4], [5]), local area wireless networks (WLANs) [6], and broadband wireless systems [2]. The basic idea is to split the signal to be modulated (usually a sequence of quadrature amplitude modulation (QAM) or phase-shift keying (PSK) symbols) into a large number of subchannels by a serial-to-parallel $(\mathrm{S} / \mathrm{P})$ conversion, and modulate them by orthogonal subcarriers. This is an effective way to reduce intersymbol interference (ISI) caused by multipaths.

In order to do either channel equalization, echo cancellation, or coherent detection, the channel needs to be estimated. To aid this, the so-called cyclic prefix (CP) is often used [7]. Different estimation approaches are available in the literature; see, e.g., [8]-[16]. The CP consists of a block of redundant samples at the beginning of each transmitted frame. It can be shown that if the length of the CP is larger than the tap size of the channel, the channel can be modeled with a constant diagonal matrix. As

Paper approved by Y. Li, the Editor for Wireless Communication Theory of the IEEE Communications Society. Manuscript received July 21, 2003; revised June 24,2004 . This paper was presented in part at the IEEE International Conference on Communications, 2003.

D. Marelli is with the Laboratoire d'Analyze, Topologie et Probabilités, Centre de Mathématiques et d'Informatique, Université de Provence, 13453 Marseille, France (e-mail: marelli@cmi.univ-mrs.fr).

M. Fu is with the School of Electrical Engineering and Computer Science, University of Newcastle, N.S.W. 2308, Australia (e-mail: eemf@ecemail. newcastle.edu.au).

Digital Object Identifier 10.1109/TCOMM.2005.858671 a consequence, the estimation algorithms are computationally very efficient. However, the $\mathrm{CP}$ consumes channel capacity.

As mentioned above, the CP needs to be ideally larger than the tap size of the channel. However, a long $\mathrm{CP}$ would take up a big capacity overhead. To avoid using a long $\mathrm{CP}$, the so-called timedomain equalizer (TEQ) is often placed immediately after the channel; see, e.g., [17]-[22]. (A variant of this method is called per-tone equalization (PTEQ) and uses a frequency-domain equivalent of the TEQ [23]). The TEQ is intended to make the combined channel-TEQ impulse response length less than or equal to the $\mathrm{CP}$ length. The main drawback of this method is that it requires a significant amount of computation, which tends to undermine the advantage of the CP.

Subband identification is a system-identification technique proposed for applications where the system to be identified can be modeled as a finite-impulse response (FIR) system of a large tap size [24], [25]. It is shown in [26] that this technique can achieve the same performance as the classical (sometimes called fullband) identification technique, but with a significantly smaller computational cost. The subband approach has also been used for a number of equalization problems; see, e.g., [27] and [28].

In this paper, we use the subband-identification technique to propose a channel-estimation method for a DMT or OFDM channel. This method has two advantages: 1) it does not consume channel capacity; and 2) it is computationally more efficient than the TEQ method when the channel impulse response is longer than the $\mathrm{CP}$.

In this paper, the channel estimation using $\mathrm{CP}$ (with or without TEQ) will be referred to as cyclic prefix estimation (CPE), whereas the method using the subband approach will be referred to as subband estimation (SBE). In our framework, we assume that a training signal is used for estimation purposes.

There are two main approaches to channel estimation.

CE1) Design an optimal [e.g., minimum mean-square error (MMSE)] estimator of the channel, based on statistical knowledge of the channel and the signals (see, e.g., [8]).

CE2) Identify the channel with an adaptive algorithm (see, e.g., [11]).

The CE1 approach is computationally more efficient, since it does not involve an adaptive algorithm. However, it relies on the assumption that the channel can be accurately described by a statistical model, and it is often arguable whether this assumption is realistic. For this reason, we will use the CE2 approach in this paper. However, the results of this paper can be easily adapted to the CE1 approach. 


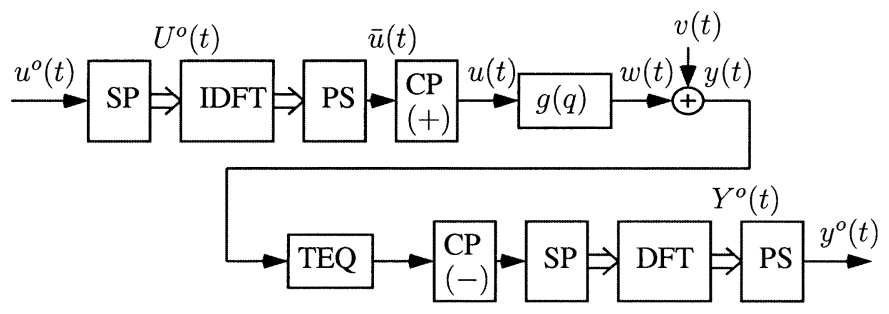

Fig. 1. DMT or OFDM scheme.

The rest of this paper is organized as follows. Section II gives an overview of DMT and OFDM systems. Section III introduces some relevant results on subband identification. Section IV studies the proposed SBE method. Section V applies the SBE method to derive a subband equalizer. Some simulation results are presented in Section VII, and concluding comments are given in Section VIII.

Throughout the paper, we use the following notation. We express scalar signals using the lowercase and vector signals using the uppercase. The superscript $(\cdot)^{o}$ denotes a baseband signal. Finally, $\hat{x}(t)$ denotes the estimate of the signal $x(t)$, and $\tilde{x}(t)=x(t)-\hat{x}(t)$, the estimation error signal.

\section{DMT AND OFDM SYSTEMS}

\section{A. Modulation Method}

In the DMT and OFDM modulation methods, the source signal $u^{o}(t)$ is split into $C$ subchannels $U^{o}(t)=$ $\left[u^{o}(t C), u^{o}(t C-1), \ldots, u^{o}(t C-(C-1))\right]^{T}$ by an $\mathrm{S} / \mathrm{P}$ conversion, and these subchannel signals are modulated by orthogonal subcarriers. The modulated signal $\bar{u}(t)$ is given by

$$
\bar{u}(t)=\sum_{c=0}^{C-1} u^{o}(k C-c) e^{j 2 \pi \frac{c}{C} t}, \quad(k-1) C<t \leq k C
$$

which can be generated by using a $C$-point inverse discrete Fourier transform (IDFT) or inverse fast Fourier transform (IFFT). The signal is transmitted in frames of $C$ symbols. Since the frame rate is $1 / C$ th of the symbol rate, ISI due to delay spreads is significantly reduced.

The complete DMT or OFDM transmission and receiving scheme is depicted in Fig. 1. Here, $g(q)$ is the discrete-time transfer function of the equivalent baseband channel, assumed to be a finite impulse response (FIR) with tap size $n_{g}$. The signal $v(t)$ is the equivalent baseband noise. The boxes denoted by SP and $\mathrm{PS}$ are serial-to-parallel and parallel-to-serial converters, respectively. The boxes denoted by $\mathrm{CP}(+)$ and $\mathrm{CP}(-)$ have the functions of adding and removing the $\mathrm{CP}$, respectively, and the block denoted by TEQ is the time-domain equalizer.

\section{B. Cyclic Prefix Channel Estimation}

Consider Fig. 1, where $Y^{o}(t)$ is defined in a way similar to $U^{o}(t)$. We denote $U^{o}(t)=\left[U_{1}^{o}(t), \ldots, U_{C}^{o}(t)\right]^{T}$ and use a similar decomposition for $Y^{o}(t)$. Suppose for a moment that the TEQ is not present. It can be shown that [7]

$$
Y^{o}(t)=G(q) U^{o}(t)+V^{o}(t)
$$

where the linear time-invariant system $G(q)$ is an equivalent model for the channel (including all the blocks between $U^{o}(t)$
TABLE I

\begin{tabular}{|c|c|}
\hline Method & $\begin{array}{c}\text { Computational Cost } \\
\text { [complex multiplications per symbol] }\end{array}$ \\
\hline CPE+TEQ1 & $9 n_{g}$ \\
\hline CPE+TEQ2 & $9\left(n_{z}+n_{p}+1\right)$ \\
\hline SBE & $\begin{array}{cl}26+\log _{2} D, & \text { if } n_{\hat{G}}=1 \\
16+\log _{2} D+2\left(9 n_{\hat{G}}-5\right), & \text { if } n_{\hat{G}} \neq 1\end{array}$ \\
\hline
\end{tabular}

Computational Cost of THE DifFERENT METHODS

and $Y^{o}(t)$ ), and $V^{o}(t)$ is the noise component of $Y^{o}(t)$. As mentioned above, the CP is used to suppress the ISI. The idea is to add, at the beginning of each frame of $C$ samples, a block of $P$ samples, which equals the last $P$ samples of the same frame. It is shown that if $P$ is greater than or equal to the impulse-response length of the channel (i.e., $P \geq n_{g}$ ), then $G(q)$ becomes a constant diagonal matrix $G(q)=\operatorname{diag}\left\{G_{c} ; c=1, \ldots, C\right\}$. In this case, $G(q)$ can be estimated by a diagonal parametric model $\hat{G}(q, \theta)=\operatorname{diag}\left\{\theta_{c} ; c=1, \ldots, C\right\}$. For the purposes of this paper, we consider the least squares (LS) channel-estimation criterion, i.e., each of the $C$ subchannel gains, $\theta_{c} ; c=1, \ldots, C$, is estimated by minimizing the power of the difference $Y_{c}^{o}(t)-\theta_{c} U_{c}^{o}(t)$. Once all the $\theta_{c}$ are computed, we can form

$$
\tilde{W}^{o}\left(t, \theta^{o}\right)=(G(q)-\hat{G}(q, \theta)) U^{o}(t) .
$$

Modulating $\tilde{W}^{o}(t, \theta)$ as in (1), the resulting signal, denoted by $\tilde{w}(t, \theta)$, represents the channel estimation error. The power of this signal is used to indicate the performance of the channel estimation.

Since $C \gg n_{g}$ typically, the method above essentially uses over-parametrization. For this reason, low-rank estimators of $G(q)$ have been proposed in the literature [8], [29].

The CPE is computationally very efficient, since it only involves the adaptive estimation of one parameter per subchannel. The computational cost is given in Table I.

\section{Time-Domain Equalizer}

As mentioned above, if $n_{g}>P$, a TEQ is placed in cascade with the channel (see Fig. 1). There are two main techniques to design the TEQ [18], [19].

TEQ1) This technique is also called optimal shortening in [18]. The impulse response of the TEQ, $\mathbf{s}(t)$, is assumed to be a FIR filter of tap size $n_{s}$. Assuming that the channel impulse response $\mathrm{g}(t)$ for $g(q)$ is known, $\mathbf{s}(t)$ is designed to minimize the energy of the shortened impulse response $\mathbf{g}_{s}(t)=(\mathbf{s} * \mathbf{g})(t)$ corresponding to those taps after the $\mathrm{CP}$ length $P$, i.e.,

$$
\mathbf{s}(t)=\arg \min _{\sigma} \sum_{t=P}^{\infty}\left|\mathbf{g}_{\sigma}(t)\right|^{2} .
$$

Hence, the knowledge of $\mathbf{g}(t)$ is required. To evaluate the computational complexity of this method, we only consider that of the identification of $\mathbf{g}(t)$, since the construction of $\mathbf{s}(t)$ is done only once, at the end of the adaptation process. 


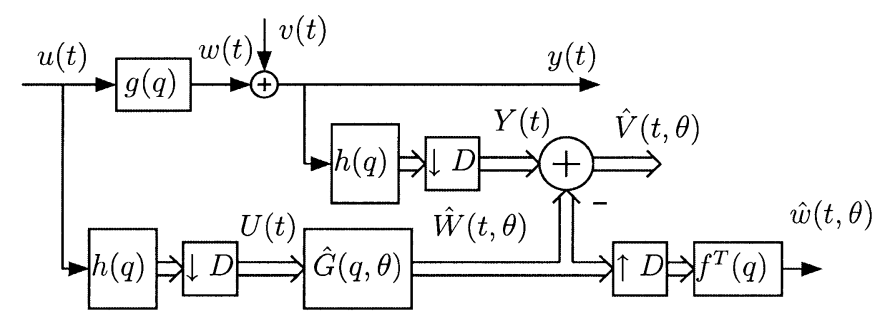

Fig. 2. Subband identification.

TEQ2) This technique is also known as LS shortening in [18]. The channel transfer function $g(q)$ is identified with a zero-pole model $r(q)=z(q) / p(q)$, where the order of $z(q)$ is less than or equal to the CP length $P$. Then, the transfer function $s(q)$ of the TEQ is chosen as $s(q)=p(q)$. If $r(q)$ is a good approximation of $g(q)$, then the shortened transfer function $g_{s}(q)$ is given by

$$
g_{s}(q)=s(q) g(q)=p(q) g(q) \simeq p(q) \frac{z(q)}{p(q)}=z(q)
$$

whose impulse response is less than or equal to $P$. The polynomials $z(q)$ and $p(q)$ have orders $n_{z}$ and $n_{p}$, respectively, and their parameters are jointly optimized in a LS sense.

The computational cost of the two methods above are also given in Table I. These methods will be compared with our new equalization method. However, we note there are modifications of TEQ1 and TEQ2 based on the bit-rate maximizing criterion [20]-[22].

\section{SUbBand IDENTIFICATION METHOD}

The scheme of the subband identification method is depicted in Fig. 2. The idea is to split both signals $u(t)$ and $y(t)$ (which will be called fullband signals) into $M$ subbands using two identical analysis filterbanks with transfer function $h(q)=\left[h_{1}(q), \ldots, h_{M}(q)\right]^{T}$. These subband signals are down-sampled, by a factor of $D$ (i.e., one every $D$ samples is taken). The results are denoted by two vector signals $U(t)=\left[U_{1}(t), \ldots, U_{M}(t)\right]^{T}$ and $Y(t)=\left[Y_{1}(t), \ldots, Y_{M}(t)\right]^{T}$. The subband parametric model is given by

$$
\hat{G}(q, \theta)=\operatorname{diag}\left\{\hat{G}_{m}\left(q, \theta_{m}\right), \quad m=1, \ldots, M\right\}
$$

where $\hat{G}_{m}\left(q, \theta_{m}\right)$ are FIR models of tap size $n_{\hat{G}}$. Its output is denoted by

$$
\hat{W}(t, \theta)=\left[\hat{W}_{1}\left(t, \theta_{1}\right), \ldots, \hat{W}_{M}\left(t, \theta_{M}\right)\right]^{T}
$$

and the prediction error defined by

$$
\hat{V}(t, \theta)=\left[\hat{V}_{1}\left(t, \theta_{1}\right), \ldots, \hat{V}_{M}\left(t, \theta_{M}\right)\right]^{T}=Y(t)-\hat{W}(t, \theta) .
$$

Each subband model $\hat{G}_{m}\left(q, \theta_{m}\right)$ is tuned to minimize the power of $\hat{V}_{m}\left(t, \theta_{m}\right)$. An up-sampler (with $D-1$ zeroes inserted between two samples) and a synthesis filterbank $f(q)=\left[f_{1}(q), \ldots, f_{M}(q)\right]^{T}$ are used to reconstruct $\hat{w}(t, \theta)$, which is an estimate of the channel output $w(t)$. The reconstruction of the signal $\hat{w}(t, \theta)$ is only required to evaluate the performance of the SBE and is not needed for the actual implementation of the method.

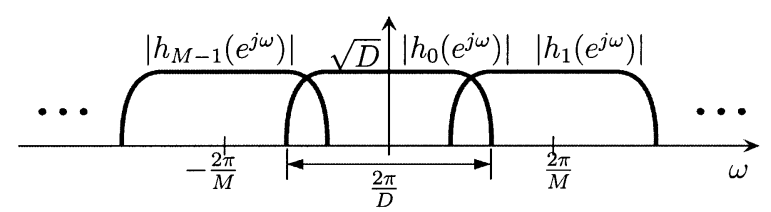

Fig. 3. Ideal filterbank. The shape of the transition bands is proportional to $\sqrt{\omega}$.

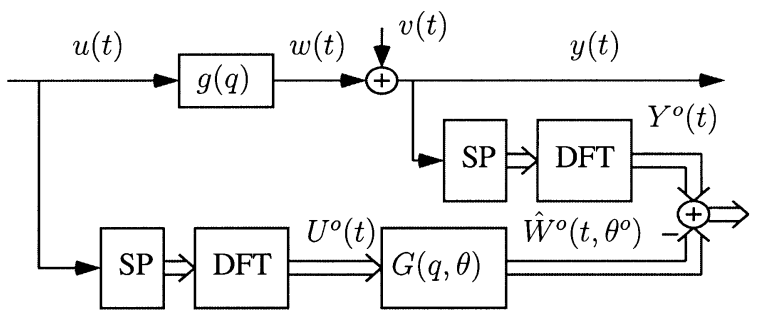

Fig. 4. Equivalent DMT or OFDM diagram.

In [26], it was shown that with a careful choice of design parameters (number of subbands $M$, downsampling factor $D$, filterbanks, and subband models), the performance of the subband method, as measured by asymptotic residual error, asymptotic convergence rate, and computational cost, can be optimized. We summarize some of these results below. Denote the power of the signal $\tilde{w}(t, \theta)=w(t)-\tilde{w}(t, \theta)$ by $S_{\tilde{w}}(\theta)$, which is a measure of the subband modeling error, by $\hat{G}(q, \theta)$. Let $\theta_{N}$ denote the set of parameters computed, by a given optimization criterion, up to time $N$. Then, the asymptotic residual error is defined as

$$
S_{\tilde{w}, \lim }=\lim _{N \rightarrow \infty} S_{\tilde{w}}\left(\theta_{N}\right)
$$

and the convergence rate is defined as

$$
\Delta(N)=\mathcal{E}\left\{S_{\tilde{w}}\left(\theta_{N}\right)-S_{\tilde{w}}\left(\theta_{N-1}\right)\right\} .
$$

In order to identify $g(q)$ with a diagonal subband model and be able to achieve $S_{\tilde{w}, \lim }=0$ with maximum convergence rate, the filterbanks $h(q)$ and $f(q)$ need to take the shapes in Fig. 3. These filters have zero gain in their stop-band. Therefore, they have an infinite (noncausal) impulse response (IIR), and need to be approximated. A computationally efficient approximation technique is presented in the Appendix. If we use critical sampling (i.e., $D=M$ ), the transition bands of the filters become zero. As a consequence, the quality of the approximation will be poor, and therefore $S_{\tilde{w}, \text { lim }}$ will be large. Hence, oversampling $(D<M)$ is used to reduce $S_{\tilde{w}, \text { lim }}$.

In order to achieve $S_{\tilde{w}, \lim }=0$, not only the filterbanks need to be IIR, but also the subband models $\hat{G}_{m}\left(q, \theta_{m}\right)$ need to have infinite parameters. A bound of $S_{\tilde{w}, \lim }$ as a function of $n_{\hat{G}}$ is given in [26]. It turns out that, in practice, $S_{\tilde{w}, \text { lim }}$ can be kept reasonably low by choosing [30]

$$
n_{\hat{G}}=\left\lceil\frac{n_{g}}{D}\right\rceil
$$

where $\lceil x\rceil$ denotes the smallest integer larger than $x$.

\section{Subband Channel Estimation}

We propose to estimate the DMT or OFDM channel using the subband-identification method. We first provide the motivation for the proposed approach. 


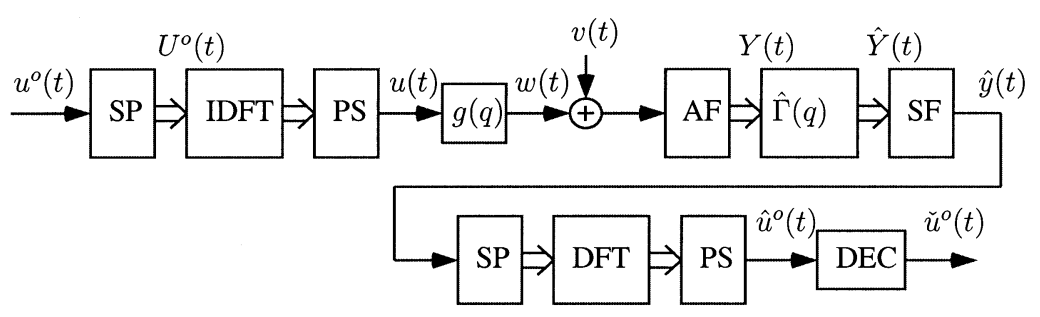

Fig. 5. Subband equalizer.

Consider the CPE method without using either CP or TEQ. An equivalent diagram of this method is given in Fig. 4. In this case, the CPE method can be thought as the subband method with $D=M=C$, with the DFTs acting as analysis filterbanks, and with subband models consisting of one tap (i.e., they are unknown constants). In this case, $D \gg n_{g}$, and the mentioned over-parametrization of the CPE can be seen from (3). The DFT acts as a filterbank, and is far from the decoupling filterbank in Fig. 3. Hence, the model $\hat{G}(q, \theta)$ is far from diagonal. This generates the need for CP. As mentioned in Section II-B, the CP length $P$ needs to be larger than $n_{g}$, for $\hat{G}(q, \theta)$ to be diagonal. When $n_{g}$ becomes larger than $P$, the transfer matrix $\hat{G}(q, \theta)$ becomes nondiagonal and nonconstant (i.e., more than one tap is needed for each subband model) again, and for this reason the TEQ is needed. So we can think of the CP and TEQ as two techniques to diagonalize $\hat{G}(q, \theta)$.

Due to the flexibility of the subband method, we are free to choose the filterbanks, $D, M$, and $n_{\hat{G}}$. If we choose the filterbanks as in Fig. 3, the subband model $\hat{G}(q, \theta)$ becomes diagonal automatically without the need of CP and TEQ. Also, the overparametrization can be easily avoided by choosing [see (3)]

$$
D \simeq n_{g} \quad \text { and } \quad n_{\hat{G}}=1
$$

As mentioned earlier, the number of subbands $M$ is chosen larger than $D$ in order to improve the approximation of the ideal filterbank of Fig. 3. It turns out that

$$
M=2 D
$$

is usually a good choice.

To evaluate the complexity of this method, we note that the subband version of the training signal $u(t)$ can be computed offline, since it is a known signal. Then, if $M$ is chosen as in (4), and for an arbitrary value of $n_{\hat{G}}$, the computational cost of the SBE has the expression given in Table I.

With the SBE so designed, the subband model $\hat{G}(q, \theta)$ is a constant diagonal matrix with no over-parametrization and no need of CP and TEQ. If $n_{g}<P$, the CPE is expected to have zero estimation error (due to the $\mathrm{CP}$ ) with a computational cost smaller than that of the SBE, since, in this case, the TEQ is not required. If $n_{g}>P$, simulation results show that the computational cost of the SBE is much lower than that of the CPE with TEQ for compatible estimation errors.

Another advantage of the SBE is that due to its computational simplicity, it is easy to increase its precision by having a minor increase in computations. As mentioned above, for the SBE to have zero estimation error, the subband models need to have $n_{\hat{G}} \rightarrow \infty$. The criterion (3) for choosing $n_{\hat{G}}$ is a practical choice for a "reasonable" precision. If the precision of the SBE needs to be increased, the product $D n_{\hat{G}}$ needs to be increased. From Table I, we can see that the computational cost is linear in $n_{\hat{G}}$ but logarithmic in $D$. So it is computationally more efficient to increase $D$ instead of $n_{\hat{G}}$.

As mentioned in the Introduction, another variant of the TEQ technique is the so-called PTEQ [23]. In this technique, the TEQ, which is assumed to be a FIR filter of tap size $n_{s}$, is moved to the frequency domain. The resulting equalizer consists of one $n_{s}$-tap equalizer per subchannel, which has $C$ successive FFTs of the received signal as its inputs for each DMT or OFDM frame (recall that $C$ denotes the DMT or OFDM frame length). It is said in [23] that the PTEQ method has a better performance than the TEQ method in terms of bit-error rate (BER), based on the argument that the equalization is done on a per-subchannel basis. However, the computational complexity of the PTEQ method is slightly greater than that of the TEQ method. The SBE method also handles equalization in the frequency domain, but the subband structure can be designed to have a single tap instead of $n_{s}$-tap subband models. In this way, the SBE method is computationally more efficient than the PTEQ method.

\section{EQUALIZER DESIGN}

As explained in Section I, the estimated subband model $\hat{G}(q, \theta)$ can be used to design a channel equalizer. The equalization scheme is shown in Fig. 5, where the boxes denoted by $\mathrm{AF}$ and $\mathrm{SF}$ are the analysis and synthesis filterbanks, respectively.

Next, we propose a formula for the subband equalizer $\hat{\Gamma}(q)$. Assuming that $\hat{G}(q, \theta)$ is an accurate subband model of $g(q)$, the $m$ th subband can be modeled as follows:

$$
Y_{m}(t)=\hat{G}_{m}\left(q, \theta_{m}\right) U_{m}(t)+V_{m}(t) .
$$

Suppose a diagonal subband equalizer is used in Fig. 5, i.e.,

$$
\hat{\Gamma}(q)=\operatorname{diag}\left\{\hat{\Gamma}_{m}(q), m=1, \ldots, M\right\} .
$$

Suppose that the $m$ th subband equalizer $\hat{\Gamma}_{m}(q)$ is optimized to minimize the power of $U_{m}(t)-\hat{\Gamma}_{m}(q) Y_{m}(t)$. Then, $\hat{\Gamma}_{m}(q)$ is given by the Wiener formula [31]

$$
\hat{\Gamma}_{m}(\omega)=\frac{\hat{G}_{m}^{*}\left(\omega, \theta_{m}\right) \Phi_{U_{m}}(\omega)}{\left|\hat{G}_{m}\left(\omega, \theta_{m}\right)\right|^{2} \Phi_{U_{m}}(\omega)+\Phi_{V_{m}}(\omega)}
$$

where $\Phi_{U_{m}}(\omega)$ and $\Phi_{V_{m}}(\omega)$ denote the power spectra of the signals $U_{m}(t)$ and $V_{m}(t)$, respectively. So we propose (5)-(6) as the design method for the subband equalizer.

If the signals $u(t)$ and $v(t)$ are white, or have the same power spectra up to a constant, then, in every subband 
$\Phi_{V_{m}}(\omega) / \Phi_{U_{m}}(\omega)=\lambda / \mu$, where $\mu$ and $\lambda$ denote the power of the signals $u(t)$ and $v(t)$, respectively. In this case

$$
\begin{aligned}
\hat{\Gamma}_{m}(\omega) & =\frac{\hat{G}_{m}^{*}\left(\omega, \theta_{m}\right)}{\left|\hat{G}_{m}\left(\omega, \theta_{m}\right)\right|^{2}+\lambda / \mu} \\
& =\left(\hat{G}_{m}\left(\omega, \theta_{m}\right)+\frac{\lambda}{\mu} \frac{1}{\hat{G}_{m}^{*}\left(\omega, \theta_{m}\right)}\right)^{-1} .
\end{aligned}
$$

It can be seen from (7) that if $n_{\hat{G}}=1$, the tap size $n_{\hat{\Gamma}}$ of the subband equalizers $\hat{\Gamma}_{m}(\omega)$ is also one, i.e., $n_{\hat{\Gamma}}=1$. In this case, the equalizer is simply a gain and is given by

$$
\hat{\Gamma}_{m}(q)=\frac{\theta_{m}^{*}}{\left|\theta_{m}\right|^{2}+\lambda / \mu}
$$

\section{Vi. Computational Cost Analysis}

In this section, we provide expressions for the computational costs of the methods involved in this paper. All the estimation methods considered involve the adaptive optimization of some set of parameters. For comparative purposes, we assume that the adaptation is done using a reasonably efficient recursive least squares (RLS) algorithm in [32, Table 6.2, p. 358] called the fast RLS algorithm (version A). For this algorithm, the amount of complex multiplications per iteration is $9 n-5$, where $n$ is the number of parameters to optimize. However, if only one parameter needs to be optimized, the RLS algorithm requires five complex multiplications per iteration. Another algorithm used is the FFT/IFFT. The computational cost of an $F$-point FFT/IFFT is $(F / 2) \log _{2} F$; see [33]. The computational cost of the fast filterbanks implementation of the Appendix depends on the design parameter $E$. As a practical criterion for this paper, we take $E=16 D$. In this case, each fast filterbank requires $4+\log _{2} D+6(M / D)$ multiplications per fullband sample.

Using the expressions above, we can deduce the computational costs of all the methods, which are summarized in Table I.

\section{SimULATION}

In order to evaluate the performance of the SBE, we compare its estimation errors with that of the CPE. The CPE is combined with either of the two impulse-response-shortening techniques, TEQ1 or TEQ2. We call these methods CPE-TEQ1 and CPE-TEQ2, respectively. We consider a DMT system of frame length $C=512$ and CP length $P=32$, where the transmitted signal $u^{o}(t)$ is a sequence of 4-QAM symbols. This resembles the forward channel of the DMT-based ADSL, as described in [17]. For design purposes, the channel tap size is assumed to be $n_{g}=100$. For the SBE, we try three different values of $D$, i.e., $D=128,256,512$. For each value of $D$, we chose $M=2 D$ and $n_{\hat{G}}=1$, as pointed out in Section IV. We parameterize the methods CPE-TEQ1 and CPE-TEQ2 to have estimation errors comparable to that of the SBE with $D=128$. For the CPE-TEQ1, we choose $n_{s}=120$, and for the CPE-TEQ2, we choose $n_{z}=32$ and $n_{p}=69$. As pointed out is Section II-B, since $C \gg n_{g}$, the CPE method needs to be compensated for over-parametrization. To this end, we used the approach introduced in [29, eq. (6)], i.e., the obtained set of frequency-domain

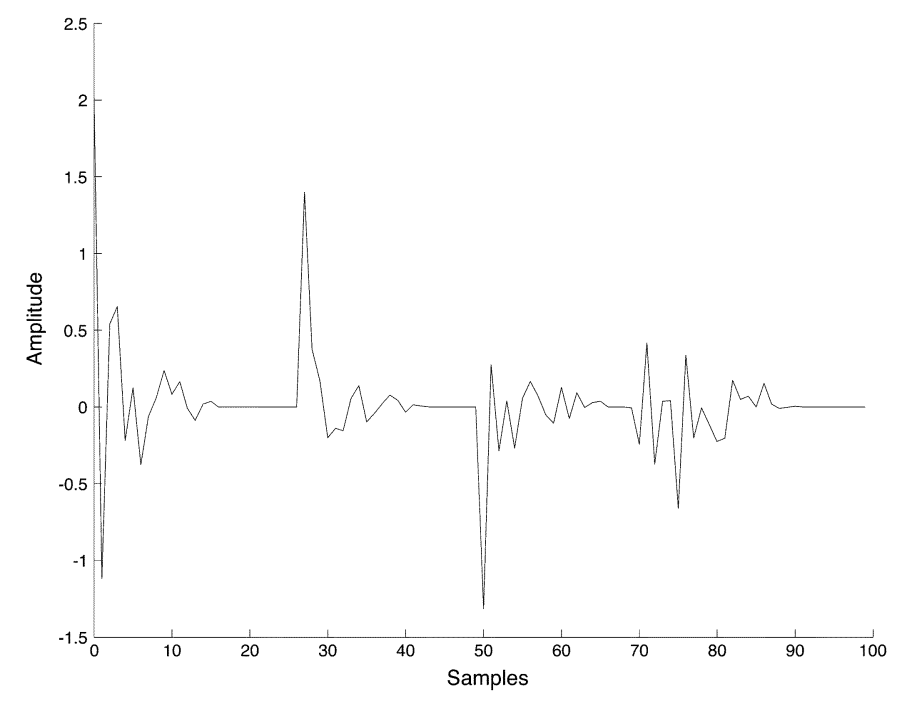

Fig. 6. Example of a 100-tap channel with 4 paths.

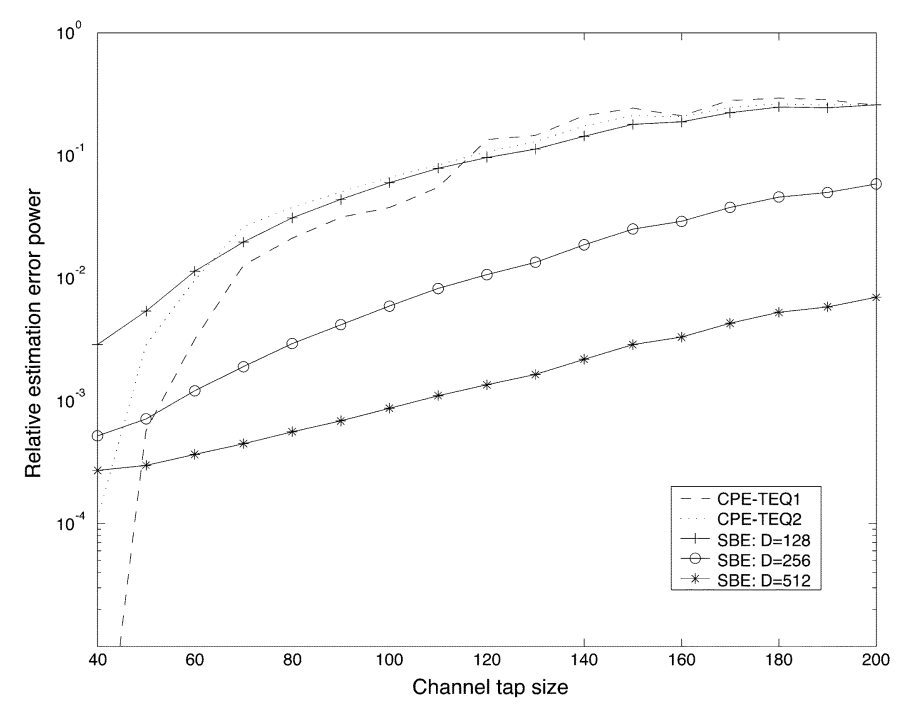

Fig. 7. Comparison of the relative estimation errors.

parameters $\left\{\theta_{c} ; c=1, \ldots, C\right\}$ is projected onto the subspace of parameters corresponding to FIR systems of tap size smaller than or equal to $n_{g}$.

The assumption $n_{g}=100$ is only for design purposes. The actual channel tap size $n_{g}^{*}$ is chosen arbitrarily. For each value of $n_{g}^{*}$, we generate a random channel, consisting of one main path and three reflections (multipaths). An example of a channel with $n_{g}^{*}=100$ is shown in Fig. 6 .

In the first simulation, we compare the estimation error of the methods in the absence of noise. This assumption is valid, since the noise level will only affect the speed of convergence of the adaptive estimation algorithm, but will not modify the final result. In order to guarantee the convergence of the algorithms, we use 20 frames for estimation. We proceed as follows. We take $n_{g}^{*}$ from 40 to 200 . For each value of $n_{g}^{*}$, we generate 100 channels. For each estimation method, a normalized estimation-error power (i.e., the estimation-error power divided by the power of the input signal) of the 100 channels are averaged. The results are shown in Fig. 7. 
TABLE II

COMPUTATIONAL COST OF THE DifFERENT METHODS

\begin{tabular}{|c|c|c|c|c|c|}
\hline Method & CPE-TEQ1 & CPE-TEQ2 & \multicolumn{3}{|c|}{ SBE } \\
\hline $\begin{array}{c}\text { Comp. Cost } \\
\text { per symbol }\end{array}$ & 900 & 900 & $\begin{array}{c}D=128 \\
33\end{array}$ & $\begin{array}{c}D=256 \\
34\end{array}$ & $\begin{array}{c}D=512 \\
35\end{array}$ \\
\hline
\end{tabular}

We can see that for small values of $n_{g}^{*}$, CPE-TEQ1 and CPETEQ2 have a smaller error than SBE. This is expected because of the use of CP. However, for $n_{g}^{*}=100$ (which equals the value of $n_{g}$ assumed for the design of the methods) and higher values, CPE-TEQ1, CPE-TEQ2, and SBE $(D=128)$ have comparable estimation errors. Also, for higher values of $D, \mathrm{SBE}$ outperforms CPE-TEQ1 and CPE-TEQ2. The advantage of the SBE is seen in the computational complexity, which is shown in Table II. The computational costs are computed using Table I. Note that for CPE-TEQ1, the cost does not include that for computing $s(t)$.

Recall that the computational cost of the SBE is logarithmic in $D$. Therefore, as shown in the table above, the SBE can be made very accurate with a minor increase in the computations.

It has to be remarked that even if the value of $n_{g}^{*}$ is made arbitrarily small, the SBE has an apparent lower bound in the estimation error of about $3 \times 10^{-4}$. This is due to the nonideal filterbanks used for subband decomposition. The approximation technique used for the simulation is the one presented in the Appendix. If higher precisions are required, the approximation needs to be improved with more computations.

In the next simulation, we analyze the equalization properties in the presence of noise. We consider a channel tap size of $n_{g}^{*}=n_{g}=100$, and again, we average over 100 channels. In this case, as the presence of the noise will slow down the convergence rate, we use 40 frames for estimation. Then, we use the estimated model to design an equalizer. The SBE-based equalizer is designed as explained in Section V. The CPE-based equalizer consists of a diagonal matrix $\hat{\Gamma}(q)=\operatorname{diag}\left\{\hat{\Gamma}_{c} ; c=1, \ldots, C\right\}$, which has as its input the signal $Y^{o}(t)$ (see Fig. 1). The gains $\hat{\Gamma}_{c}$ are given by

$$
\hat{\Gamma}_{c}=\frac{\left(\theta_{c}\right)^{*}}{\left|\theta_{c}\right|^{2}+\frac{\lambda^{o}}{\mu^{o}}}
$$

where $\mu^{o}$ and $\lambda^{o}$ denote the power of the signals $u^{o}(t)$ and $v^{o}(t)$, respectively, and $v^{o}(t)$ is the noise component of the received signal $y^{o}(t)$. The received signal after equalization is passed through a 4-QAM detector. In Fig. 8, we show the BER as a function of $E_{b} / N_{0}$ (i.e., the ratio of the transmitted energy per bit and the noise power spectral density).

We can see that the SBE-based equalization method with $D=128$ has a performance somewhat better than that of the two CPE-based equalization methods, whereas for larger values of $D$, it outperforms them much more, especially at high $E_{b} / N_{0}$.

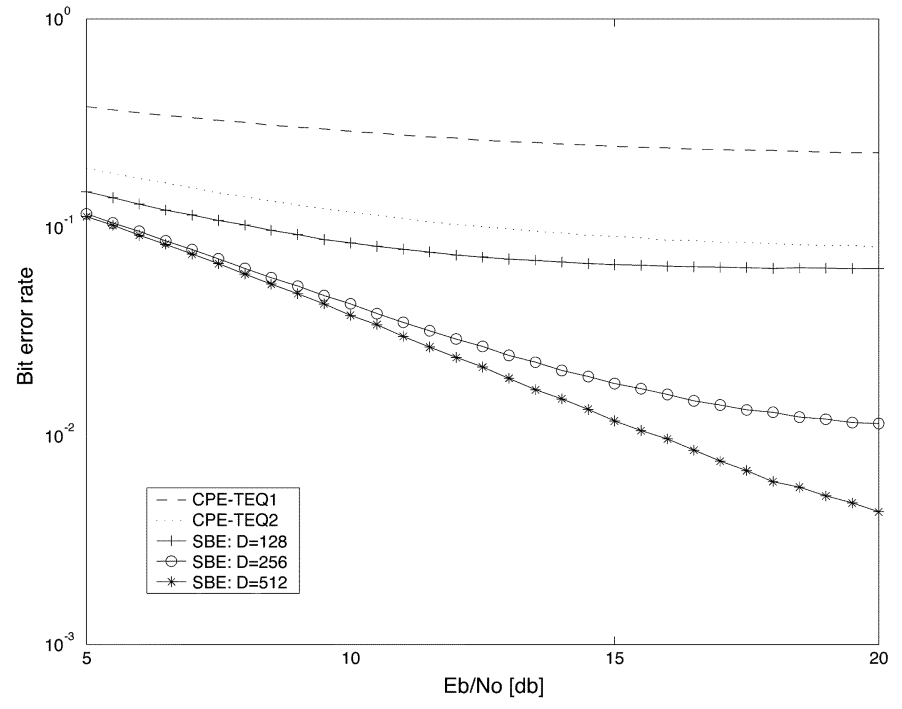

Fig. 8. Comparison of the BERs for a channel tap size $n_{g}=50$.

\section{CONCLUSION}

In this paper, we have proposed a subband approach to channel estimation and equalization for DMT and OFDM systems. Conceptually, the main advantage of the approach is that it maintains the spirit of the CP-based equalization without the use of CP and TEQ. More precisely, the basic idea of CP-based equalization is to transform the DMT or OFDM signals, through DFT and the removal of CP, into a set of signals where the effect of the channel on each signal is a simple static gain which can be easily estimated or equalized. The subband approach achieves a similar purpose by replacing the DFT with an optimized filterbank and appropriate subband structure.

Application-wise, the main advantages of the subband approach are the following.

1) Better capacity use is achieved because of the removal of the CP.

2) If the $\mathrm{CP}$ can be made comparable to the channel impulse response so that the TEQ is not required, then the CPE has smaller estimation error, compared with the SBE. If instead the TEQ is needed, the SBE gives a significantly smaller estimation error and BER, in comparison with the CP-based methods.

3) It is much more computationally efficient, compared with the CP-based methods.

Finally, we point out that there are many other signal-processing problems in communications where the subband approach finds applications. The reader is referred to [26]-[28], and [34]-[38] for introduction, theory, and applications of the subband approach. 


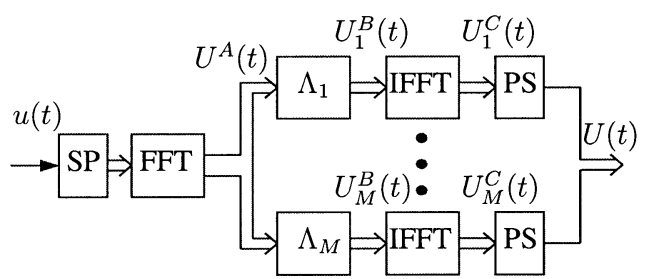

(a)

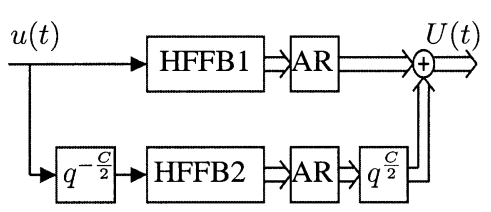

(b)

Fig. 9. (a) Half fast-filterbank. (b) Complete fast-filterbank.

\section{APPENDIX}

\section{FAST FILTERBANKS FOR SUBBAND IDENTIFICATION}

In this appendix, we introduce a computationally efficient way for approximating the filterbanks in Fig. 3, in the case where $D$ is a power of two.

In Fig. 2, the subband signals $U_{m}(t)$ are generated by downsampling the output of the filters $h_{m}(q), m=1, \ldots, M$. Then [39, eq. (4.1.13)]

$$
U_{m}\left(e^{j \omega}\right)=\frac{1}{D} \sum_{d=0}^{D-1} h_{m}\left(e^{j \frac{\omega+d}{D}}\right) u\left(e^{j \frac{\omega+d}{D}}\right)
$$

Let $\sigma_{m}$ denote the support of the frequency response of the filters $h_{m}\left(e^{j \omega}\right), m=1, \ldots, M$, (i.e., $\sigma_{m}=\{\omega \in[0,2 \pi)$ : $\left.\left.h_{m}\left(e^{j \omega}\right) \neq 0\right\}\right)$. The set $\sigma_{m}$ is connected and has a measure of $2 \pi / D$. Then, it can be shown that

$U_{m}\left(e^{j D \omega}\right)=\frac{1}{D} h_{m}\left(e^{j \omega}\right) u\left(e^{j \omega}\right), \quad \omega \in \sigma_{m}, \quad m=1, \ldots, M$.

The idea of this approximation method is the following. The precision of the approximation depends on a constant $K$ which needs to be a power of two. Then, the spectrum of the signal $u(t)$ is sampled at $E=K D$ equally spaced frequencies $\omega_{e}=$ $2 \pi(e-1 / E), e=1, \ldots, E$. For each $m \in\{1, \ldots, M\}$, we define the set of indexes $I_{m}=\left\{e: \omega_{e} \in \sigma_{m}\right\}$. Since $\sigma_{m}$ has a measure of $2 \pi / D$, then $I_{m}$ has $K$ indexes. Then, using (9), we map the $K$ frequencies $\omega_{e}$ into the spectrum of the signal $U_{m}(t)$ generating the frequencies $\nu_{e}$ which satisfy $e^{j v_{e}}=e^{j D \omega_{e}}, e \in$ $I_{m}$. Define the indexes $e_{m, k}, k=1, \ldots, K$, such that $\nu_{e_{m, k}}=$ $2 \pi(k-1 / K)$. Now, using (9), we can form the samples of the spectrum of the signals $U_{m}(t)$ by weighting the samples of the spectrum of $u(t)$, according to the frequency response of the subband filters, as follows:

$$
U_{m}\left(e^{j \nu_{e_{m}, k}}\right)=\frac{1}{D} h\left(e^{j \omega_{e_{m}, k}}\right) u\left(e^{j \omega_{e_{m}, k}}\right), \quad k=1, \ldots, K
$$

In order to implement (10), we use the scheme in Fig. 9(a), where the boxes marked with SP and PS are serial-to-parallel and parallel-to-serial converters, respectively.

A block of $E$ samples of the signal $u(t)$ is taken into the frequency domain by an $E$-point FFT, to form the signals $U^{A}(t)=\left[U_{1}^{A}(t), \ldots, U_{E}^{A}(t)\right]^{T}$. Then, the frequency-domain representation of the subband signals $U_{m}(t)$ (i.e., $\left.U_{m}^{B}(t)=\left[U_{m, 1}^{B}(t), \ldots, U_{m, K}^{B}(t)\right]^{T}\right)$ is formed by multiplying
$U^{A}(t)$ with the frequency-weighting diagonal matrix $\Lambda_{m}$, defined by

$\Lambda_{m}=\operatorname{cshiftr}\left\{\left[\operatorname{diag}\left\{\lambda_{m, k}, \quad k=1, \ldots, K\right\}, \mathbf{0}_{K \times(E-K)}\right], i_{m}\right\}$

where the matrix operator $\operatorname{cshiftr}\{A, x\}$ is the $x$-times circular shift on the right of the matrix $A, i_{m}=\min \left\{I_{m}\right\}$ and $\lambda_{m, k}=$ $(1 / D) h\left(e^{j \omega_{e}, k}\right)$. Finally, in each subband, an IFFT forms the time-domain version of $U_{m}(t)$.

With this procedure, for every block of $E$ samples of the signal $u(t)$, a block of $K$ samples of the signals $U(t)$ is generated. The problem with this approach is that the samples of $U(t)$ which are close to the block limits will be highly affected by time aliasing. In order to avoid this, we just take the $K / 2$ samples centered in the middle of the block of $K$ samples of $U(t)$, and discard the others. Then, in order to generate the missing parts of $U(t)$, another instance of the scheme of Fig. 9(a), which is shifted by half of the block length, is used. Each one of these two instances is called half fast filterbanks (HFFB). The complete scheme is shown in Fig. 9(b), where the boxes marked with HFFB1 and HFFB2 are half fast filterbanks, and the alias removal (AR) boxes represent the process of taking the $K / 2$ central samples of each block.

In order to approximate the synthesis filterbank, the map $T_{h}$ : $u(t) \mapsto U(t)$ needs to be inverted. Since $T_{h}$ is not invertible, in general, its pseudoinverse $\left(T_{h}^{+}=\left(T_{h}^{*} T_{h}\right)^{-1} T_{h}^{*}\right)$ needs be used. The implementation of $T_{h}^{+}$is computationally involved, so an approximate approach that works satisfactorily in practice is used instead. The idea is to invert every block of the procedure above, using either its inverse or its pseudoinverse. In particular, the map $\Lambda=U^{A}(t) \mapsto\left[U_{1}^{B}(t), \ldots, U_{M}^{B}(t)\right]$ needs to be taken as a block where, since it is not invertible, its pseudoinverse $\Lambda^{+}$ need to be applied.

In order to find an expression for $\Lambda^{+}$, let $\Lambda=$ $\left[\Lambda_{1}^{T}, \ldots, \Lambda_{M}^{T}\right]^{T}$, with $\Lambda_{m}$ defined as in (11). Then

$$
\Lambda^{+}=\left(\Lambda^{*} \Lambda\right)^{-1} \Lambda^{*}=\left[\Lambda_{1}^{+}, \ldots, \Lambda_{M}^{+}\right]
$$

where $\Lambda_{m}^{+}$are the $E \times K$ matrices given by

$$
\begin{aligned}
& \Lambda_{m}^{+}=\operatorname{cshiftr}\left\{\left[\operatorname{diag}\left\{\frac{\lambda_{m, k}^{*}}{\sum_{i=1}^{M}\left|\lambda_{i, k}\right|^{2}}, k=1, \ldots, K\right\},\right.\right. \\
& \left.\left.\mathbf{0}_{K \times(E-K)}\right]^{T}, i_{m}\right\}
\end{aligned}
$$


Summary of the Algorithm: The input data of the algorithm are $M$ and $D$, where $D$ is a power of two. The constant $K$ is chosen according to the desired accuracy of the approximation and is also a power of two. The algorithm consists of two HFFBs.

\section{HFFB1:}

1) Starting from sample $t=0$, do an $\mathrm{S} / \mathrm{P}$ conversion of $u(t)$ by splitting it in blocks of $E=K D$ consecutive samples.

2) Do a $E$-point FFT of each block, to get the signal $U^{A}(t)=\left[U_{1}^{A}(t), \ldots, U_{E}^{A}(t)\right]^{T}$.

3) For each $m \in\{1, \ldots, M\}$, define $U_{m}^{B}(t)=\Lambda_{m} U^{A}(t)$, where $\Lambda_{m}$ is given by (11).

4) For each $m \in\{1, \ldots, M\}$, define $U_{m}^{C}(t)=$ $\left[U_{m, 1}^{C}(t), \ldots, U_{m, K}^{C}(t)\right]^{T}$ as the IFFT of $U_{m}^{B}(t)$.

5) For each $m \in\{1, \ldots, M\}$, set $U_{m, k}^{C}(t)=0$, for $k \notin$ $\{(1 / 4) K, \ldots,(3 / 4) K\}$.

6) For each $m \in\{1, \ldots, M\}$, do a parallel-to-serial conversion of $U_{m}^{C}(t)$ to form $U_{m}(t)$.

7) The subband (vector) signal generated by HFFB1 is given by $U^{(1)}(t)=\left[U_{1}(t), \ldots, U_{M}(t)\right]^{T}$.

\section{HFFB2:}

1) Repeat step 1) of HFFB1, but starting from sample $t=$ $E / 2$.

2) Repeat steps 2) to 7) of HFFB1 to form the subband (vector) signal $U^{(2)}(t)$.

3) The final subband (vector) signal is given by $U(t)=$ $U^{(1)}(t)+U^{(2)}(t)$.

\section{REFERENCES}

[1] J. Bingham, "Multicarrier modulation for data transmission: An idea whose time has come," IEEE Commun. Mag., vol. 28, no. 5, pp. 5-14, Dec. 1990.

2] R. van Nee and R. Prasad, OFDM for Wireless Multimedia Communications. Boston, MA: Artech House, 2000.

[3] J. L. J. Cimini, "Analysis and simulation of a digital mobile channel using orthogonal frequency division multipexing," IEEE Trans. Commun., vol. COM-33, no. 7, pp. 665-675, Jul. 1985.

[4] T. Starr, J. M. Cioffi, and P. J. Silverman, Understanding Digital Subscriber Line Technology. Englewood Cliffs, NJ: Prentice-Hall PTR, 1998.

[5] J. Chow, J. Tu, and J. Cioffi, "A discrete multitone transceiver system for HDSL applications," IEEE J. Sel. Areas Commun., vol. 9, no. 6, pp. 895-908, Aug. 1991.

[6] R. van Nee, G. Awater, M. Morikura, H. Takanashi, M. Webster, and K. Halford, "New high-rate wireless LAN standards," IEEE Commun. Mag., vol. 37, no. 12, pp. 82-88, Dec. 1999.

[7] Z. Wang and G. B. Giannakis, "Wireless multicarrier communications," IEEE Signal Process. Mag., vol. 17, no. 3, pp. 29-48, May 2000.

[8] O. Edfors, M. Sandell, J.-J. van de Beek, S. K. Wilson, and P. O. Brjesson, "OFDM channel estimation by singular value decomposition," IEEE Trans. Commun., vol. 46, no. 7, pp. 931-939, Jul. 1998.

[9] Y. Li, "Pilot-symbol-aided channel estimation for OFDM in wireless systems," IEEE Trans. Veh. Technol., vol. 49, no. 4, pp. 1207-1215, Jul. 2000

[10] Y. Baoguo, C. Zhigang, and K. Letaief, "Analysis of low-complexity windowed DFT-based MMSE channel estimator for OFDM systems," IEEE Trans. Commun., vol. 49, no. 11, pp. 1977-1987, Nov. 2001

[11] W. Xiaowen and K. R. Liu, "Adaptive channel estimation using cyclic prefix in multicarrier modulation system," IEEE Commun. Lett., vol. 3 , no. 10, pp. 291-293, Oct. 1999.

[12] R. W. Heath, Jr. and G. Giannakis, "Exploiting input cyclostationarity for blind channel identification in OFDM systems," IEEE Trans. Signal Process., vol. 47, no. 3, pp. 848-856, Mar. 1999.
[13] M. Morelli and U. Mengali, "A comparison of pilot-aided channel estimation methods for OFDM systems," IEEE Trans. Signal Process., vol. 49, no. 12, pp. 3065-3073, Dec. 2001.

[14] V. Mignone and A. Morello, "CD3-OFDM: A novel demodulation scheme for fixed and mobile receiver," IEEE Trans. Commun., vol. 44 no. 9, pp. 1144-1151, Sep. 1996.

[15] M. Speth, S. A. Fechtel, G. Fock, and H. Meyr, "Optimum receiver design for wireless broadband systems using OFDM-Part 1," IEEE Trans. Commun., vol. 47, no. 11, pp. 1668-1677, Nov. 1999.

[16] J. K. Cavers, "An analysis of pilot-symbol-assisted modulation for Rayleigh-fading channels," IEEE Trans. Veh. Technol., vol. 40, no. 4, pp. 686-693, Nov. 1991.

[17] M. Ho, J. Cioffi, and J. Bingham, "Discrete multitone echo cancellation," IEEE Trans. Commun., vol. 44, no. 7, pp. 817-825, Jul. 1996.

[18] P. Melsa, R. Younce, and C. Rohrs, "Impulse response shortening for discrete multitone transceivers," IEEE Trans. Commun., vol. 44, no. 12 , pp. 1662-1672, Dec. 1996.

[19] Y. Changchuan and Y. Guangxin, "Optimal impulse response shortening for discrete multitone transceivers," Electron. Lett., vol. 34, no. 1, pp. 35-36, Jan. 1998.

[20] N. Al-Dhahir and J. Cioffi, "Optimum finite-length equalization for multicarrier transceivers," IEEE Trans. Commun., vol. 44, no. 1, pp. 56-64, Jan. 1996.

[21] W. Henkel and T. Kessler, "Maximizing the channel capacity of multicarrier transmission by suitable adaptation of the time-domain equalizer," IEEE Trans. Commun., vol. 48, no. 12, pp. 2000-2004, Dec. 2000

[22] G. Arslan, B. Evans, and S. Kiaei, "Equalization for discrete multitone transceivers to maximize bit rate," IEEE Trans. Signal Process., vol. 49, no. 12, pp. 3123-3135, Dec. 2001.

[23] K. V. Acker, G. Leus, M. Moonen, O. van de Wiel, and T. Pollet, "Pertone equalization for DMT-based systems," IEEE Trans. Commun., vol 49, no. 1, pp. 109-119, Jan. 2001.

[24] Y. Itoh, Y. Maruyama, N. Furuya, and T. Areseki, "Acoustic echo canceller for teleconference," in Proc. Int. Conf. Commun., 1985, pp. $1498-1502$.

[25] W. Kellermann, "Kompensation akustischer echos in frequenzteilbaendern" (in German), Frequent, vol. 39, pp. 209-215, Jul.-Aug. 1985.

[26] D. Marelli and M. Fu, "Performance analysis for subband identification," IEEE Trans. Signal Process., vol. 51, no. 12, pp. 3128-3142, Dec. 2003

[27] S. Weiss, M. Rupp, and L. Hanzo, "A fractionally spaced DFE with subband decorrelation," in Conf. Rec. 34th Asilomar Conf. Signals, Syst., Comput., 2000, pp. 1767-1771.

[28] H. Mohamad, S. Weiss, M. Rupp, and L. Hanzo, "Fast adaptation of fractionally spaced equalizers," Electron. Lett., vol. 38, no. 2, pp. 96-98, Jan. 2002.

[29] L. Deneire, P. Vandenameele, L. van der Perre, B. Gyselinckx, and M Engels, "A low-complexity ML channel estimator for OFDM," IEEE Trans. Commun., vol. 51, no. 2, pp. 135-140, Feb. 2003.

[30] J. P. Reilly, M. Wilbur, M. Seibert, and N. Ahmadvand, "The complex subband decomposition and its application to the decimation of large adaptive filtering problems," IEEE Trans. Signal Process., vol. 50, no. 11, pp. 2730-2743, Nov. 2002.

[31] T. Kailath, A. H. Sayed, and B. Hassibi, Linear Estimation. Englewood Cliffs, NJ: Prentice-Hall, 2000.

[32] J. G. Proakis, C. M. Raider, F. Ling, and C. L. Nikias, Advanced Dig ital Signal Processing. New York: Maxwell Macmillan International, 1992.

[33] G. Strang, Linear Algebra and Its Applications, 3rd ed. San Diego, CA: Harcourt Brace Jovanovich, 1988.

[34] J. J. Shynk, "Frequency-domain and multirate adaptive filtering," IEEE Signal Process. Mag., vol. 9, no. 1, pp. 14-37, Jan. 1992.

[35] A. Gilloire and M. Vetterlli, "Adaptive filtering in subbands with critical sampling: Analysis, experiments, and application to acoustic echo cancellation," IEEE Trans. Signal Process., vol. 40, no. 8, pp. 1862-1875, Aug. 1992

[36] Y. Lu and J. Morris, "Gabor expansion for adaptive echo cancellation," IEEE Signal Process. Mag., vol. 16, no. 2, pp. 68-80, Mar. 1999.

[37] C. Breining, P. Dreiseitel, E. Hansler, A. Mader, B. Nitsch, H. Puder, T. Schertler, G. Schmidt, and J. Tilp, "Acoustic echo control, an application of very-high-order adaptive filters," IEEE Signal Process. Mag., vol. 16, no. 4, pp. 42-69, Jul. 1999.

[38] D. Marelli and M. Fu, "Asymptotic properties of subband identification," IEEE Trans. Signal Process., vol. 52, no. 1, pp. 142-154, Jan. 2004.

[39] P. Vaidyanathan, Multirate Systems and Filterbanks. Englewood Cliffs, NJ: Prentice-Hall, 1993. 


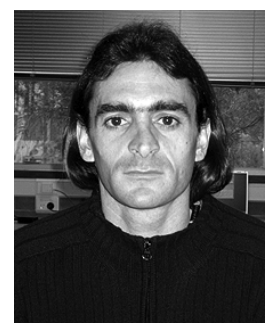

Damián Marelli received the Bachelor's degree in electronics engineering from the Universidad Nacional de Rosario, Rosario, Argentina, in 1995, and the Ph.D. degree in electrical engineering and the Bachelor's (Hons. 1) degree in mathematics from the University of Newcastle, Newcastle, Australia, in 2003.

He was a Software Engineer with Tesis Ingeniería Informática S.R.L., Rosario, Argentina, from 1995 to 1996, and with BLC S.A., Rosario, Argentina, in 1998. In 1997 he held a Teaching and Research Assistantship with the Universidad Nacional de Rosario. In 2003, he held a Research Associate position with the School of Electrical Engineering and Computer Science, University of Newcastle. During 2004, he held a Postdoctoral Research Fellowship with the Laboratoire d'Analyze Topologie et Probabilités, CNRS/Université de Provence, Provence, France, and since 2005, he holds a combined Teaching and Reseach position with the Ecole Généraliste d'Ingénieurs de Marseille, Marseille, France. His main research interests include multirate signal processing, time-frequency analysis, system identification, and statistical signal processing.

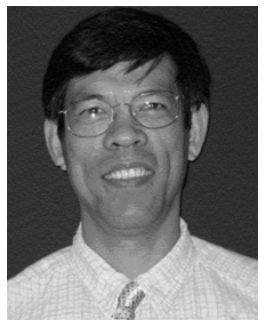

Minyue Fu (S'84-M'87-SM'94-F'04) received the Bachelor's degree in electrical engineering from the University of Science and Technology of China, Hefei, China, in 1982, and the M.S. and Ph.D. degrees in electrical engineering from the University of Wisconsin, Madison, in 1983 and 1987, respectively.

From 1983 to 1987, he held a Teaching Assistantship and a Research Assistantship with the University of Wisconsin, Madison. He was a Computer Engineering Consultant with Nicolet Instruments, Inc., Madison, WI, during 1987. From 1987 to 1989 , he served as an Assistant Professor with the Department of Electrical and Computer Engineering, Wayne State University, Detroit, MI. For the summer of 1989, he was with the Universite Catholique de Louvain, Louvain, Belgium, as a Maitre de Conferences Invited. He joined the Department of Electrical and Computer Engineering, University of Newcastle, Newcastle, Australia, in 1989. He served as the Head of the Department of Electrical and Computer Engineering from 1998 to 2002. Currently, he is a Chair Professor in Electrical Engineering and the Head of the School of Electrical Engineering and Computer Science. In addition, he was a Visiting Associate Professor at the University of Iowa, Ames, in 1995-1996, and a Senior Fellow/Visiting Professor at Nanyang Technological University, Singapore, 2002. His main research interests include control systems, signal processing, and communications. Currently, he is an Area Editor for the Journal of Optimization and Engineering and Associate Editor for Automatica.

Dr. Fu served as an Associate Editor for the IEEE TRANSACTIONS ON AUTOMATIC CONTROL for four years. 